\title{
UNCERTAINTY EVALUATION VIA FUZZY ENTROPY FOR MULTIPLE FACTS
}

\author{
Sanghyuk Lee \\ Xi'an Jiaotong-Liverpool University \\ 111 Ren'ai Road, Suzhou Dushu Lake, HET, Jiangsu Province, \\ 215123, China \\ Sanghyuk.Lee@xjtlu.edu.cn \\ T.O. Ting \\ Xi'an Jiaotong-Liverpool University \\ 111 Ren'ai Road, Suzhou Dushu Lake, HET, Jiangsu Province, \\ 215123, China \\ toting@xjtlu.edu.cn
}

\section{ABSTRACT}

The fuzzy entropy designed for multiple facts selection has been carried out in this work. The entropy for the fuzzy data with respect to a specified fact is designed through a distance measure method. The obtained fuzzy entropy is then applied for the selection from multiple facts. From the relevant fuzzy entropy, it is concluded that data uncertainty information is limited by the total fact of $n-1$. The bounded calculation of data uncertainty to each fact is proven for multiple facts, and the decision of fuzzy data to the certain fact among multiple facts has been considered with the assistance of fuzzy entropy calculation.

Keywords: Multiple Facts, Fuzzy Entropy, Decision Making, Similarity Measure

\section{INTRODUCTION}

Generally, numerical data are defined with fixed characteristic function values that satisfy either one or zero. Hence, the ordinary data shows the fact with full certainty. However, uncertainty is a common phenomenon in the actual data. Particularly, circumstances are often represented by heuristic expressions such as "high", "average", and "low". Hence, a mathematical tool is essential to express heuristic objects into a mathematical definition. Hence, the study on data with a degree of membership function has been carried out, pioneered by Zadeh ${ }^{1}$. From his description of fuzzy sets, data is 
explained through certainty and uncertainty simultaneously because of membership function, i.e., flexible characteristic functions in mathematical notation. Numerous researches have been rigorously carried out on fuzzy sets, such as control, signal processing, modeling, or other related fields ${ }^{2,3}$.

Recently, studies of data uncertainties for fuzzy data have been continuously investigated by many researchers ${ }^{4,5}, 6$. With the help of Shannon's information theory ${ }^{7}$, De Luca and Termini defined entropy definition on fuzzy sets ${ }^{8}$. Liu also proposed that the relations between entropy and similarity measure with distance measure ${ }^{4}$. Hence, fuzzy entropy calculation can be applied to the evaluation of certainty or uncertainty. The considered fuzzy entropy is emphasized on the calculation of uncertainty with respect to the corresponding fact. Application results are found in our previous work on reliable data selection ${ }^{9}$.

Generally, in order to obtain decision or detection for multi facts over the same domain, it will require a more appropriate measure to calculate the certainty or uncertainty to the corresponding facts. Fuzzy entropy is also applicable to multi-fact circumstances; multi-facts are regarded as the variation of the corresponding ordinary sets. For widely distributed data, it is somehow vague on the deterministic meaning of the fact. Hence, it is required to evaluate uncertainties for target data with respect to the corresponding deterministic fact(s). In our previous results, fuzzy entropies were designed and studied. The relations between the fuzzy entropy and the similarity measure were also discussed ${ }^{10}$. This fuzzy entropy can be formulated through similarity measure and vice versa.

Decision among multiple facts that are distributed over whole range can be explained by which fact is the closest to the considered data. Due to the distributed data on multi-facts of the same universe of discourse that contains confusion in selection, decision measure is needed to solve this category of problem. The problem is solved through the evaluation of uncertainty and evaluation value is obtained by employing the entropy on fuzzy sets. Conventional entropy was designed for evaluation of uncertainty of fuzzy data with respect to the single fact. In this study, by extending the conventional result, total uncertainty to the multi facts is also obtained, and the upper limit is also calculated. The total entropy indicates the data uncertainty itself, that is, the crisper the data, the smaller the entropy value.

In the following section, fuzzy entropy is introduced to describe the quantization of uncertainty. The conventional fuzzy entropies which were mathematically driven are also explained by the graphical illustration. Further, properties of entropy are also included. In Section 3, the decision procedure for fuzzy data with respect to the multi facts is formulated. Besides, the total uncertainties with respect to the multi facts are also 
formulated in this section. Fuzzy entropy upper limit is derived, which depends on the number of considered facts. Finally, conclusions are given in Section 4.

\section{ENTROPY MEASURE FOR UNCERTAINTY EVALUATION}

It is well-known that the fuzzy entropy describes the degree of fuzziness with respect to the corresponding numerical data. Zadeh proposed the concept of fuzzy entropy in $1968^{1}$. However, his definition did not satisfy the four axioms of the fuzzy entropy definition; it was just suggested as a kind of Shannon information entropy. De Luca and Termini defined information entropy about fuzzy sets as follows in the mapping ${ }^{8}$ :

$$
\begin{gathered}
H: \xi \rightarrow R^{+} \\
A \mid \rightarrow H(A)
\end{gathered}
$$

where $\xi(X)$ is a set consisting of all fuzzy subsets of universe of discourse $X$, and $A \in \xi(X)$. The following properties were illustrated by De Luca and Termini, which made it more convenient to understand the concept of fuzzy entropy for the fuzzy set $A \in \xi(X)$.

E1. $H(A)=0$ if and only if $\mu_{A}(x)=0$ or $1, \forall x \in X$

E2. $H(A)$ takes the maximum value if and only if $\mu_{A}(x)=1 / 2, \forall x \in X$

E3. If $A \leq B$ then $H(A)=0$, where $A \leq B$ means $A$ is a sharp set $B$,

E4. $H(A)=H(\bar{A})$, where $\bar{A}$ is a complementary set of $A$

Kaufmann also proposed the information entropy of fuzzy sets. However the fuzzy set poses a problem when the membership degree is the same $^{11}$. Further, Kosko and Pal proposed fuzzy entropies which satisfied De Luca and Termini's fuzzy entropy ${ }^{12,13}$. There are many fuzzy entropy definitions. We also derived our own definition in previous results ${ }^{14,15}$. The entropy of fuzzy data sets with respect to the corresponding ordinary set can be designed using distance measure.

$$
\begin{aligned}
& e\left(A, A_{\text {near }}\right)=d\left(A \cap A_{\text {near }},[1]_{X}\right)+d\left(A \cup A_{\text {near }},[0]_{X}\right)-1 \\
& e\left(A, A_{\text {near }}\right)=d\left(A, A \cap A_{\text {near }}\right)+d\left(A_{\text {near }}, A \cap A_{\text {near }}\right)
\end{aligned}
$$

where ordinary set or crisp set $A_{\text {near }}$ represents the crisp set "near" to the fuzzy set $A$. $A_{\text {near }}$ is referred by variable as $0 \leq$ near $\leq 1$. For example, the membership value of crisp set $A_{0.5}$ represents one when $\mu_{A}(x) \geq 0.5$, and it is zero when $\mu_{A}(x) \leq 0.5$ where $A \cap B$ and $A \cup B$ are expressed the minimum and maximum value among $A$ and $B$; expressions are commonly used in fuzzy set theory. The distance is defined by $d(A, B)=$ 
$\frac{1}{n} \sum_{i=1}^{n}\left|\mu_{A}\left(x_{i}\right)-\mu_{B}\left(x_{i}\right)\right| .[1]_{X}$ and $[0]_{X}$ satisfy one and zero for the whole universe of discourse, respectively. They all represent the degree of uncertainty between fuzzy set and corresponding deterministic ordinary set $A_{\text {near. }}$. By the analysis of (1) and (2), it can also be stated that fuzzy entropies represent the dissimilarity measure (DM) between sets $A$ and $A_{\text {near }}$ where $P(X)$ and $F(X)$ are the ordinary set and fuzzy set, respectively. [1/2 $]_{\mathrm{X}}$ is the fuzzy set in which the value of the membership function is $1 / 2 . A^{c}$ denotes the complement of fuzzy set $A \forall A \in F(X)$. Fuzzy entropy implies the degree of uncertainty or the dissimilarity between two data sets, fuzzy set and corresponding ordinary set. Hence, it can be designed through many ways satisfying the fuzzy entropy definition such as the difference or differential area between set $A$ and corresponding ordinary data set $A_{\text {near }}$. The smaller the difference is in value, the closer the degree of similarity between two sets. Naturally, a small entropy value guarantees less uncertainty of the fuzzy set to the corresponding ordinary set.

\section{APPLICATIONS OF FUZZY ENTROPY AND SIMILARITY MEASURE TO DECISION MAKING}

Considering fuzzy set $\mathrm{A}$ and corresponding membership function $\mu_{A}(x), x \in X$ are distributed over several facts such as $F_{1}, F_{2}, \cdots F_{N}$. By calculating and comparing the entropies between fuzzy set $\mathrm{A}$ and facts, $\mathrm{F}_{1}, \mathrm{~F}_{2}, \cdots \mathrm{F}_{\mathrm{N}}$, the least uncertain pair can be selected. By obtaining a minimum value such as $\min _{i} e\left(A, F_{i}\right)$, we determine which fact among $\mathrm{F}_{1}, \mathrm{~F}_{2}, \cdots \mathrm{F}_{\mathrm{N}}$ is the closest to fuzzy set A. If paire $\left(A, F_{n}\right)$ is satisfied by minimum value, fuzzy set $\mathrm{A}$ will have the least uncertainty to the fact $F_{n}$. When multiple facts are assumed as the disjoint facts, that is, if $F_{i} \cap F_{j}=0$ for $i \neq j$, and supports of facts $\mathrm{F}_{1}, \mathrm{~F}_{2}, \cdots \mathrm{F}_{\mathrm{N}}$ constitute the whole universe of discourse. Next, we consider two facts case, by extending the entropy of the fuzzy set with respect to two facts, fuzzy entropy to the corresponding fact (1) is considered as the following formulation:

$$
e\left(A, F_{M}\right)=d\left(A \cap F_{M},[1]_{X}\right)+d\left(A \cup F_{M},[0]_{X}\right)-1 \text {, for } M=1,2 .
$$

Two facts $F_{1}$ and $F_{2}$, and data distribution set A over the whole universe of discourse are illustrated in Figure 1. 


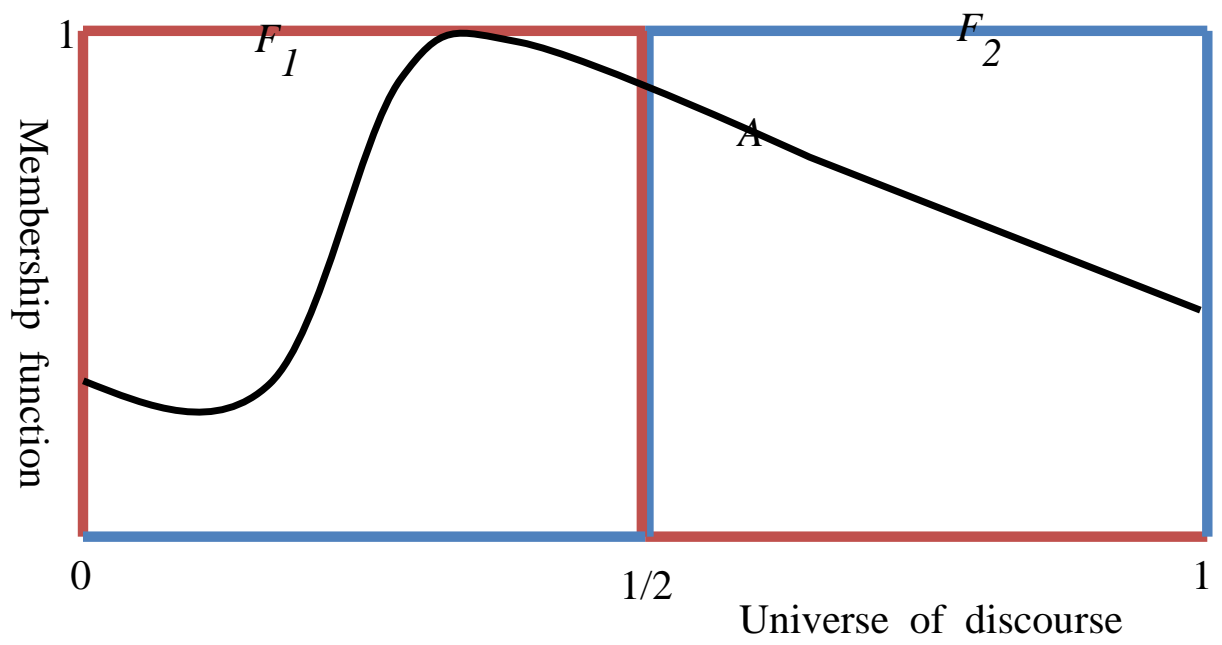

Figure 1. Fuzzy set $A$ and corresponding two facts $F_{1}$ and $F_{2}$

It can be easily shown that the summation of entropies between fuzzy set $A$ and the corresponding facts $F_{1}$ and $F_{2}$ satisfies the following property.

Proposition 3.1 For the two facts over the whole universe of discourse, the following property is satisfied.

$$
\sum_{i=1}^{2} e\left(A, F_{i}\right)=1
$$

Proof: By graphical explanation it is obvious. However, it is also clear to prove the relation

$$
e\left(A, F_{1}\right)+e\left(A, F_{2}\right)=1 \text { or } e\left(A, F_{1}\right)=1-e\left(A, F_{2}\right) .
$$

For disjoint two facts, $F_{1}=F_{2}{ }^{c}$ or $F_{2}=F_{1}{ }^{c}$ has to be satisfied.Hence, summation of the fuzzy entropy measure (2) satisfies

$$
\begin{gathered}
e\left(A, F_{1}\right)+e\left(A, F_{2}\right)=e\left(A, F_{1}\right)+e\left(A, F_{1}{ }^{c}\right) \\
=d\left(A, A \cap F_{1}\right)+d\left(F_{1}, A \cap F_{1}\right)+d\left(A, A \cap F_{1}{ }^{c}\right)+d\left(F_{1}{ }^{c}, A \cap F_{1}{ }^{c}\right) .
\end{gathered}
$$

Entropy summation with respect to two facts (4) will be verified if (5) satisfies one. It is convenient to divide the universe of discourse as $\mu_{\mathrm{F}_{1}}(\mathrm{x})=1$ and $\mu_{\mathrm{F}_{1}}(\mathrm{x})=0$. The mentioned two intervals are the same when $A \cap F_{1}=$ Aand $\cap F_{1}=[0]_{X}$. For $A \cap F_{1}=A$,

$$
\begin{gathered}
d\left(A, A \cap F_{1}\right)+d\left(F_{1}, A \cap F_{1}\right)+d\left(A, A \cap F_{1}{ }^{c}\right)+d\left(F_{1}{ }^{c}, A \cap F_{1}{ }^{c}\right) \\
=d\left(F_{1}, A\right)+d\left(A,[0]_{X}\right)=1 / 2
\end{gathered}
$$


is satisfied. That is, the first half of the universe of discourse, in this interval following the properties $\cap \mathrm{F}_{1}=\mathrm{A}, \mu_{\mathrm{F}_{1}}(\mathrm{x})=[1]_{\mathrm{X}}$, and $\mu_{\mathrm{F}_{1}} \mathrm{c}(\mathrm{x})=[0]_{\mathrm{X}}$ are satisfied.

where interval for $\mathrm{A} \cap \mathrm{F}_{1}=[0]_{\mathrm{X}}$,

$$
\begin{gathered}
d\left(A, A \cap F_{1}\right)+d\left(F_{1}, A \cap F_{1}\right)+d\left(A, A \cap F_{1}{ }^{c}\right)+d\left(F_{1}{ }^{c}, A \cap F_{1}{ }^{c}\right) \\
=d\left(A,[0]_{X}\right)+d\left(F_{1}{ }^{c}, A\right)=1 / 2
\end{gathered}
$$

is also satisfied with respect to $\mathrm{A} \cap \mathrm{F}_{1}=[0]_{\mathrm{X}}, \mu_{\mathrm{F}_{1}}(\mathrm{x})=[0]_{\mathrm{X}}, \mu_{\mathrm{F}_{1}} \mathrm{c}(\mathrm{x})=$ $[1]_{\mathrm{X}}$. Therefore (4) is satisfied.

Proposition 3.2 For $\mathrm{n} \geq 3$ multi facts as over the whole universe of discourse, the following property is satisfied.

$$
\sum_{i=1}^{n} e\left(A, F_{i}\right) \leq n-1
$$

Proof: With (2) the following expression is followed

$$
\sum_{i=1}^{n} e\left(A, F_{i}\right)=\sum_{i=1}^{n}\left\{d\left(A, A \cap F_{i}\right)+d\left(F_{i}, A \cap F_{i}\right)\right\} \text {,for } n \geq 3 .
$$

Similarly with the proof of Proposition 3.1, division of $A \cap F_{1}=A$ and $A \cap$ $F_{1}=[0]_{X}$ is also available.For the interval of $A \cap F_{1}=A$, that is, $\mu_{F_{1}}(x)=[1]_{X}$, then

$$
\sum_{i=1}^{n} e\left(A, F_{i}\right)=\sum_{i} d\left(F_{i}, A \cap F_{i}\right)=\sum_{i} d\left(F_{i}, A\right)
$$

is satisfied because $d\left(F_{1}, A \cap F_{1}\right)$ is zero. Therefore (7) represents area between 1 and Afor $\forall \mathrm{x} \in \mathrm{X}$ when $\mu_{\mathrm{F}_{1}}(\mathrm{x})=[1]_{\mathrm{X}}$; that is the area between $[1]_{\mathrm{X}}$ and $\mathrm{A}^{\mathrm{c}}$.

As for the case of $A \cap F_{i}=[0]_{X}$, this means that the fuzzy set is placed outside of $\mathrm{F}_{\mathrm{i}}$, for $\mathrm{i}=1,2, \cdots \mathrm{n}$. Therefore, $d\left(F_{i}, A \cap F_{i}\right)=$ $\mathrm{d}\left(\mathrm{F}_{\mathrm{i}},[0]_{\mathrm{X}}\right)=0$ is satisfied. Naturally,

$$
\sum_{i=1}^{n} e\left(A, F_{i}\right)=\sum_{i} d\left(A, A \cap F_{i}\right)=\sum_{i} d\left(A,[0]_{X}\right)
$$

is followed. It means $n-1$ times area of $A$. Hence, $\sum_{\mathrm{i}=1}^{\mathrm{n}} \mathrm{e}\left(\mathrm{A}, \mathrm{F}_{\mathrm{i}}\right)$ represents $\mathrm{n}-1$ times area of $A$ plus area of $A^{c}$. Finally,

$$
\sum_{i=1}^{n} e\left(A, F_{i}\right)=\text { Whole area }+(n-1) \text { times of area } A \leq(n-1)
$$

is obtained. Therefore (6) is satisfied.

\section{ILLUSTRATIVE EXAMPLE}

The entropy calculations over the multiple facts are carried using an example in ${ }^{9}$. The random selection problem for 65 student marks is done. In 
this case, student grades are normally distributed with the mean mark of 52.7 and standard deviation of 14.49. Using the selection principles, four selections are carried in four trials. Three grades $F_{1}$ to $F_{3}$ are assigned by evaluations from mark zero to one hundred (full score). Grade " $F_{1}$ " represents 70 to 100 marks, $F_{2}$ is assigned for mark ranges from 35 to 69, and $F_{3}$ for the remaining marks. Three classes are disjointed from each other. For three facts case, the following entropy evaluation is applied:

$$
\begin{gathered}
e\left(A, F_{1}\right)+e\left(A, F_{2}\right)+e\left(A, F_{3}\right) \\
=d\left(A, A \cap F_{1}\right)+d\left(F_{1}, A \cap F_{1}\right)+d\left(A, A \cap F_{2}\right)+d\left(F_{2}, A \cap F_{2}\right)+ \\
d\left(A, A \cap F_{3}\right)+d\left(F_{3}, A \cap F_{3}\right)
\end{gathered}
$$

Table 1 indicates that the fuzzy entropy calculation of each trial to the

\begin{tabular}{|c|c|c|c|c|c|c|c|}
\hline \multirow[b]{2}{*}{ Trials } & \multicolumn{7}{|c|}{ Values } \\
\hline & Mark & $\begin{array}{c}\text { Membership } \\
\text { of } A\end{array}$ & $\begin{array}{c}\text { Membership } \\
\text { of } B\end{array}$ & $\begin{array}{c}\text { Membership } \\
\text { of } C\end{array}$ & $\begin{array}{c}\text { Entropy } \\
\left(F_{i}, A\right)\end{array}$ & $\begin{array}{c}\text { Entropy } \\
\left(F_{i}, B\right)\end{array}$ & $\begin{array}{c}\text { Entropy } \\
\left(F_{i}, C\right)\end{array}$ \\
\hline \multirow{5}{*}{$\begin{array}{l}\text { First } \\
\text { trial }\end{array}$} & 25 & 0.5 & 0.166 & 0 & & & \\
\hline & 44 & 0.12 & 0.8 & 0 & 0.124 & 0.6266 & 0.38 \\
\hline & 54 & 0 & 0.866 & 0.08 & 0.676 & 0.1734 & 0.66 \\
\hline & 61 & 0 & 0.633 & 0.22 & 0.324 & 0.693 & 0.14 \\
\hline & 80 & 0 & 0 & 0.60 & & & \\
\hline \multirow{5}{*}{$\begin{array}{l}\text { Second } \\
\text { trial }\end{array}$} & 50 & 0 & 1 & 0 & & & \\
\hline & 52 & 0 & 0.933 & 0.04 & $\underline{0}$ & 0.8464 & $\underline{0.092}$ \\
\hline & 55 & 0 & 0.833 & 0.1 & 1 & 0.1536 & 0.908 \\
\hline & 57 & 0 & 0.766 & 0.14 & $\underline{0}$ & 0.8464 & $\underline{0.092}$ \\
\hline & 59 & 0 & 0.7 & 0.18 & & & \\
\hline \multirow{5}{*}{$\begin{array}{l}\text { Third } \\
\text { trial }\end{array}$} & 43 & 0.14 & 0.766 & 0 & & & \\
\hline & 52 & 0 & 0.933 & 0.04 & $\underline{0.028}$ & 0.7528 & $\underline{0.12}$ \\
\hline & 54 & 0 & 0.866 & 0.08 & 0.972 & 0.2472 & 0.88 \\
\hline & 55 & 0 & 0.833 & 0.10 & $\underline{0.028}$ & 0.7528 & $\underline{0.12}$ \\
\hline & 69 & 0 & 0.366 & 0.38 & & & \\
\hline \multirow{5}{*}{$\begin{array}{c}\text { Fourth } \\
\text { trial }\end{array}$} & 12 & 0.76 & 0 & 0 & & & \\
\hline & 46 & 0.08 & 0.866 & 0 & 0.064 & 0.7198 & 0.196 \\
\hline & 53 & 0 & 0.9 & 0.06 & 0.736 & 0.0802 & 0.732 \\
\hline & 55 & 0 & 0.833 & 0.1 & 0.368 & 0.7198 & 0.068 \\
\hline & 91 & 0 & 0 & 0.82 & & & \\
\hline
\end{tabular}
corresponding three facts.

Table 1. Entropy calculations over three facts 
With (6) fuzzy entropy of four trials is evaluated with respect to the three facts $F_{1}, F_{2}$, and $F_{3}$. In order to calculate fuzzy entropy transform of each trial data to fuzzy membership value should be obtained in advance. We have considered fuzzy membership function with respect to the facts $F_{1}, F_{2}$, and $F_{3}$.Corresponding fuzzy sets to facts $F_{1}, F_{2}$, and $F_{3}$ are assigned as $\mathrm{A}, \mathrm{B}$ and $\mathrm{C}$, respectively. Low membership values provide a small uncertainty value, which means that it is close to the corresponding fact. Hence, first trial and fourth trial close to fact $F_{1}$, but second and third trial are not easy to be determined. For second trial, it is close to $F_{2}$, however it make confusion to decide. The main reason for this the confusion originated from the shape of membership function and complementary characteristic of fuzzy entropy definition. It represents similar result of third trial.

\section{CONCLUSIONS}

The fuzzy entropy design is carried out considering multiple facts. The distance measure calculation provides the basis for the design of this fuzzy data with respect to multi facts. The analysis on the relationship of entropy values to multiple facts is explained via graphical illustration and the result provides that data uncertainty information was limited by the total fact $(n)$ minus one $(n-1)$. Proofs are derived from two facts to the general multiple cases. The bounded calculation of data uncertainty to each fact is verified from the results on a selection problem involving student marks. Simple example was illustrated for calculation of multiple fact fuzzy entropy.

\section{REFERENCES}

[1] L.A. Zadeh, Fuzzy sets. Information and Control, 8(3), p338-353, 1965.

[2] D. Driankov, H. Hellendoorn, and M. Reinfrank, An introduction to fuzzy control. Berlin, Germany: Springer, 1996.

[3] C.C. Kung, and J.Y. Su, Affine Takagi-Sugeno fuzzy modelling algorithm by fuzzy c-regression models clustering with a novel cluster validity criterion. Control Theory \& Application, 1(5), p1255-1265, 2007. http://dx.doi.org/10.1049/iet-cta:20060415.

[4] L. Xuecheng, Entropy, distance measure and similarity measure of fuzzy sets and their relations. Fuzzy Sets and Systems, 52(3), p 305-318, 1992. http://dx.doi.org/10.1016/0165-0114(92)90239-Z.

[5] D. Bhandari, and N.R. Pal, Some new information measure of fuzzy sets. Information Science, vol. 67(3), p209-228, 1993. http://dx.doi.org/10.1016/0020-0255(93)90073-U.

[6] A. Ghosh, Use of fuzziness measure in layered networks for object extraction: A generalization. Fuzzy Sets and Systems, 72(3), p331-348, 
1995. http://dx.doi.org/10.1016/0165-0114(94)00291-E.

[7] C.E. Shannon, A mathematical theory of communication. The Bell System Technical Journal, 27(3), p379-423, 1948. http://dx.doi.org/10.1002/j.1538-7305.1948.tb01338.x.

[8] A. De Luca, and S. Termini, A definition of nonprobablistic entropy in the setting of fuzzy sets theory. Information and Control, 20(4), p301-312, 1972. http://dx.doi.org/10.1016/S0019-9958(72)90199-4.

[9] S.H. Lee, Y.T. Kim, S.P. Cheon, and S.S. Kim, Reliable data selection with fuzzy entropy. Lecture Notes in Artificial Intelligence, 3613, p203-212, 2005. http://dx.doi.org/10.1007/11539506_27.

[10] S.H. Lee, H.J. Park, and W.J. Park, Similarity computation between fuzzy set and crisp set with similarity measure based on distance. Lecture Notes in Computer Science, 4993, p644-649, 2008. http://dx.doi.org/10.1007/978-3-540-68636-1_77.

[11] A. Kaufmann, Introduction to the theory of fuzzy subsets. New York: Academic Press, 1975.

[12] B. Kosko, Neural Networks and Fuzzy Systems. Englewood Cliffs, NJ: Prentice-Hall, 1992.

[13] N.R. Pal, and S.K. Pal, Object-background segmentation using new definitions of entropy. IEE Proceedings E (Computers and Digital Techniques), 136(4), p284-295, 1989. http://dx.doi.org/10.1049/ip-e.1989.0039.

[14] S.H. Lee, W. Pedrycz, and GyoyongSohn, Design of similarity and dissimilarity measures for fuzzy sets on the basis of distance measure. International Journal of Fuzzy Systems, 11(2), p67-72, 2009.

[15] S.H. Lee, K.H. Ryu, and G.Y. Sohn, Study on Entropy and Similarity Measure for Fuzzy Set. IEICE transactions on information and systems, 92(9),

p1783-1786. http://dx.doi.org/10.1587/transinf.E92.D.1783. 
